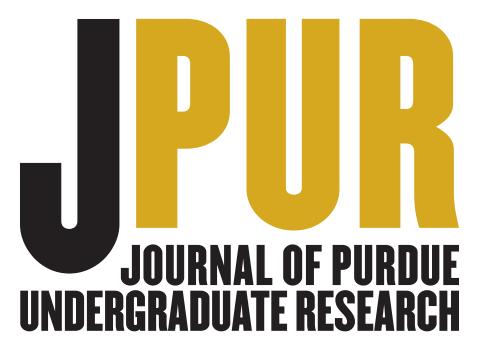

\title{
AGRICULTURE
}

\section{Tickling Rats: Differential Benefits for Pet Store Rats}

\section{Student researcher: Whitney Blankenberger, Senior}

Animal welfare and the effects of the human-animal bond are becoming increasingly important to researchers and the public. Animal use in biomedical research is indispensable and inevitably creates stressful situations for the animals. One way to mediate this stress and improve rat welfare is by using a handling technique called tickling. Tickling, which mimics rat rough-and-tumble play, reduces fear of humans and stress of injections (Cloutier \& Newberry, 2007; Cloutier, Panksepp, \& Newberry, 2012). When rats play or are tickled, they elicit ultrasonic vocalizations (USV), which cannot be heard by humans. These vocalizations can be recorded and analyzed using specialized sound equipment and software. USVs of $50 \mathrm{kHz}$ indicate positive emotions, while $20-\mathrm{kHz}$ USVs indicate negative emotions. Tickling has been shown to benefit laboratory rats, but some individuals respond more positively to tickling and may receive greater benefits. Additionally, welfare improvements have yet to be empirically validated in pet rats.

Thirty-six female pet store rats were sampled across two replicates. During each replicate, rats were randomly placed into one of three cages. One cage, designated as the control group, received standard, minimal handling throughout the study. After 3-4 days of acclimation, rats were tickled for 3 consecutive days by the researcher and their USVs were recorded. Based on the total number of positive vocalizations produced, the rats were then split into high- and low-calling cages. After 4 more days of being tickled by the employees, rats were evaluated using an unfamiliar approach and restraint test to evaluate fear behaviors and ease of restraint. We began the approach test by placing each rat in a glass tank opposite from an unfamiliar hand. After one minute had elapsed, the unfamiliar researcher picked up the rat to manually restrain it, using a technique commonly used at veterinary hospitals and in laboratories.

After 30 seconds of restraint, the rat was then placed back into the tank for an additional 1-minute approach test. These approach and restraint tests were video-recorded for later analysis by video coders blinded to the treatment groups. Reliability of the coders was tested using intraclass correlation and was maintained at a high level throughout data collection. We documented the number of restraint attempts to assess rat handleability. A restraint attempt was defined as when the researcher's first two fingers touched the animal's neck and ended when the rat was either lifted up or the fingers stopped touching the neck. The preliminary results indicate that all tickled rats required fewer restraint attempts than control rats, and low-calling rats reared less during both approach tests, a behavior indicative of comfort and exploration. These findings support previous research, which has shown that tickling rats can help alleviate their stress and improve their welfare.

\section{Research advisor Brianna Gaskill and graduate research advisor Megan LaFollette write: "Whitney has been essential to the success of our project with Petco. Our study uniquely demonstrates the positive impact of establishing a human- animal bond. Tickled rats view humans more positively, enabling them to adapt to new people and situations, and increasing the likelihood of finding a good home."}



Tickling begins with dorsal contact, followed by the handler flipping the rat and then pinning it down. This image exhibits the pin. For more photos and videos, visit https://vet.purdue.edu/ discovery/gaskill/resources.php. 